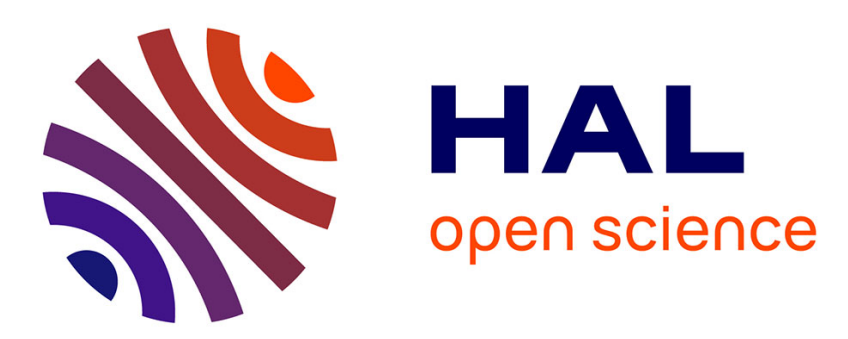

\title{
Using Fuzzy Logic to Involve Individual Differences for Predicting Cybersickness during VR Navigation
}

Yuyang Wang, Jean-Rémy Chardonnet, Frédéric Merienne, Jivka Ovtcharova

\section{To cite this version:}

Yuyang Wang, Jean-Rémy Chardonnet, Frédéric Merienne, Jivka Ovtcharova. Using Fuzzy Logic to Involve Individual Differences for Predicting Cybersickness during VR Navigation. 2021 IEEE Virtual Reality and 3D User Interfaces (VR), Mar 2021, Lisbonne, Portugal. pp.373-381, 10.1109/VR50410.2021.00060 . hal-03187239

\section{HAL Id: hal-03187239 \\ https://hal.science/hal-03187239}

Submitted on 31 Mar 2021

HAL is a multi-disciplinary open access archive for the deposit and dissemination of scientific research documents, whether they are published or not. The documents may come from teaching and research institutions in France or abroad, or from public or private research centers.
L'archive ouverte pluridisciplinaire HAL, est destinée au dépôt et à la diffusion de documents scientifiques de niveau recherche, publiés ou non, émanant des établissements d'enseignement et de recherche français ou étrangers, des laboratoires publics ou privés. 


\section{Using Fuzzy Logic to Involve Individual Differences for Predicting Cybersickness during VR Navigation}

\author{
Yuyang Wang* \\ Arts et Metiers Institute of Technology \\ LISPEN, HESAM Université, UBFC
}

\author{
Jean-Rémy Chardonnet ${ }^{\dagger}$ \\ Arts et Metiers Institute of Technology \\ LISPEN, HESAM Université, UBFC
}

Jivka Ovtcharova ${ }^{\S}$

Institute for Information Management in Engineering

Karlsruhe Institute of Technology

\author{
Frédéric Merienne \\ Arts et Metiers Institute of Technology \\ LISPEN, HESAM Université, UBFC
}

\begin{abstract}
Many studies have explored how individual differences can affect users' susceptibility to cybersickness in a VR application. However, the lack of strategy to integrate the influence of each factor on cybersickness makes it difficult to utilize the results of existing research. Based on the fuzzy logic theory that can represent the effect of different factors as a single value containing integrated information, we developed two approaches including the knowledgebased Mamdani-type fuzzy inference system and the data-driven Adaptive neuro-fuzzy inference system (ANFIS) to involve three individual differences (Age, Gaming experience and Ethnicity). We correlated the corresponding outputs with the simulator sickness questionnaire (SSQ) scores in a simple navigation scenario. The correlation coefficients obtained through a 4-fold cross validation were found statistically significant with both fuzzy logic approaches, indicating their effectiveness to influence the occurrence and the level of cybersickness. Our work provides insights to establish customized experiences for VR navigation by involving individual differences.
\end{abstract}

Index Terms: Human-centered computing-Human computer interaction (HCI) - Interaction paradigms - Virtual reality; Computing methodologies-Artificial intelligence-Knowledge representation and reasoning - Vagueness and fuzzy logic; Information systems-Information retrieval-Users and interactive retrievalPersonalization

\section{INTRODUCTION}

Consumer-friendly virtual reality (VR) gained popularity in the last years thanks to the development of affordable head-mounted displays such as HTC Vive and Oculus Rift. The easy access to these devices enables researchers and engineers to develop various VR applications for medical training [19], education [34] or patient rehabilitation [30]. VR also represents an excellent opportunity for companies to reorganize themselves and adapt their processes to new conditions imposed especially by the COVID-19 pandemic [10]. For example, Yang et al. reported that during the global pandemic, highfidelity and low-latency VR technologies helped many conferences successfully going online, featuring participants' virtual avatars fully mobile and immersed in virtual conference environments, which contributed to preventing the virus from spreading [46]. The Na-

\footnotetext{
*e-mail: yuyang.wang@ensam.eu

†e-mail: jean-remy.chardonnet@ensam.eu

‡e-mail: frederic.merienne@ensam.eu

$\S$ e-mail: jivka.ovtcharova@kit.edu
}

tional Academy of Engineering (NAE) spotlit the advantages of VR by advocating that "Enhancing Virtual Reality" is among the top grand challenges of engineering in the twenty-first century [29]. VR has attracted worldwide stakeholders to impact various sectors, and it is expected to reach a $\$ 80.7$ billion market size by 2024 [14].

The characteristic of VR is to enable a user to be immersed in a virtual environment (VE) and to interact with it [37], which means back and forth actions between the user and the VE. Consequently, the focal point of VR should be to provide a more user-oriented and customized experience for better immersion, rather than technological aspects. Similarly, Merienne claimed that the perceiver is at the center of a VR application, and awareness of the technologies and human factors are essential to promote the efficiency of the application [28].

Navigation is a fundamental task for a large number of domains using VR. The users ought to adapt their viewpoint so as to explore unknown VEs, search or maneuver objects [26]. However, navigation in a VE is associated with the possible manifestation of various negative symptoms such as nausea, disorientation, headaches, fatigue, sweating and eye strain, which is termed as cybersickness [27]. Multiple human-related factors such as sex, age, gaming experience and ethnicity, and external factors, including navigation methods and visual display parameters, have proved to influence the occurrence of cybersickness; the correlation between these factors and cybersickness has also been widely investigated in previous work [7 944$]$. The level of sickness symptoms varies significantly among individuals, depending on the technologies being utilized, the tasks being conducted and the design of the virtual environment [20]. However, in practice, controlling cybersickness based on external factors relies heavily on the collaboration between application developers and device manufacturers to optimize the display techniques, which is effective but also costly. We aim to propose a light indicator that can adapt the navigation settings based on individual characteristics, such that the application developer can maximize the use and advantage of existing devices.

The motion sickness susceptibility questionnaire (MSSQ) shortform [11] can estimate one's susceptibility to cybersickness by analyzing different sorts of motion that can induce sickness, for example, cars, buses or coaches, trains and so on. However, it fails to include the effect of individual characteristics such as Age, Gaming experience, Ethnicity among others [36]. Existing references studied the effect of individual characteristics on cybersickness [7 9 |44], while very little research investigated strategies to utilize this knowledge to design a cybersickness indicator with which we can get preliminary recognition of one's susceptibility to cybersickness beforehand. The objective of this work is to bridge this gap. Our ambition is wide, but in this paper, we present the fundamental step to develop this cybersickness indicator: we first present the approach to fuse knowledge from the current literature, based on the fuzzy inference system (FIS) and neural network algorithms, which can infer the 
quantized cybersickness indicator; next, we validate its reliability from a theoretical viewpoint. In the long term, we intend to develop a customized navigation interface by deploying such an indicator so that a VR system could better adapt to users. Our choice for FIS lies in the fact that numberless regression techniques are data-driven models that can predict individual susceptibility to cybersickness. However, they are not suitable for the current situation. Fuzzy logic models are formulated from existing rules or learned from data to formulate the rules, enabling researchers to apply expert knowledge to an application. At the same time, regression techniques are only nonlinear fitting of data correlation. Also, regression techniques rely heavily on the dataset's quality, which is a challenge and cannot make the most of prior information based on expert knowledge. We argue that prior expert knowledge, accumulated over decades of intense research, should not be dismissed and ignored.

\section{FuzZy INFERENCE Systems}

A fuzzy inference system (FIS) is a nonlinear mapping which deduces outputs according to fuzzy reasoning and a class of fuzzy if-then rules. FIS is primarily applied to situations where either the expression about the investigated problems is vague and uncertain or the systems are difficult to be exactly modeled [47]. FIS relies heavily on the knowledge and experience from professional and domain experts, and therefore it is hard to attain satisfactory outcomes if enough prior information is lacking [3. 5]. The structure of a FIS is depicted in Fig. 1. The database defines membership functions (MFs) used to partition linguistic variables (small, middle, large, and so on), and then, the inference engine carries out a reasoning procedure based on the fuzzy rules and given inputs to determine a reasonable output or consequence. In this work, as the correlation between influence factors and cybersickness can be acquainted with domain experts or past literature, FIS can include this knowledge naturally and predict cybersickness conveniently. We will detail this knowledge that we obtained from references or numerical data, and apply them to the FIS structure in Section 3

The literature reports three types of FIS: Mamdani-type, Sugenotype and Tsukamoto-type [18]. Our work here will use the Mamdanitype and the Sugeno-type models. The Mamdani-type system needs to find the centroid of a composed MF by integrating across a continuously varying function. The Sugeno-type system uses a singleton (constant value or linear mathematical functions) as the MF of the rule consequent [18]. The Mamdani method is advantageous to utilize expert knowledge in a more intuitive and human-like manner despite substantial computational burden, while the Sugeno method is computationally beneficial and works well with optimization and adaptive techniques.

On the other hand, the Adaptive neuro-fuzzy inference system (ANFIS) is a hybrid method that incorporates the benefits of both FIS and artificial neural networks (ANN) to derive the fuzzy if-then rules with suitable MFs acquired from numerical data [17]. The main advantage of ANFIS is to automatically determine the optimal parameters of FIS from data instead of using prior knowledge and information from experts or past literature. This is achieved thanks to the strong learning ability of ANN, so that optimizing the unknown parameters with the input-output data sets is workable, and the optimization of these parameters during the training process is carried out to minimize the error between the predicted output and the actual output [12]. The parameters optimized by ANFIS consist of the premise parameters that represent the shape of the MFs, and the consequent parameters that illustrate the comprehensive output of ANFIS [39]. With these optimal parameters, ANFIS can be deployed to predict or estimate the output with new inputs. Back to Fig. 1. when the fuzzy rules are constructed with experts or literature knowledge, FIS is commonly referred as Mamdani-type system, and when the fuzzy rules are derived from numerical data, FIS is noted as ANFIS.
FIS has been employed in various computer-human interface (CHI) areas to develop subject-dependent systems. Wu et al. suggested including linguistic rules instead of precise functions to model uncertainties, and also to create fuzzy rule-based controllers to describe the user's affective or cognitive state [45]. Ota et al. proposed applying rule-based fuzzy logic to measure the competence and surgical skills of the trainee of a VR educational simulator, and this evaluation can be conducted together with the teaching session [32].

There exist also many studies based on fuzzy logic to support adaptive systems. Cueva-Fernandez et al. presented an adaptive speed interface for users to create software applications in the automotive context only with their voice. The interface can suit the user's level of experience based on fuzzy rules [6]. With seven fuzzy rules considering ten characteristics, they computed a single output: the level of expertise and concentration of the user, i.e., beginner, intermediate, or advanced; then the system presents a higher or lower level of options and information depending on the user's level. Nyongesa et al. developed a fuzzy logic-based approach for webpage adaptation to improve users' shopping experience [31]. As fuzzy logic can help analyze ambiguous presentations of shopping activities, much information such as purchase frequency, last purchase time and product presentation is obtained from users to facilitate interface personalization and adaptation. Hamam et al. proposed a taxonomy for measuring a haptic VR application's experience quality based on the Mamdani-type FIS [13]. Their model contained five inputs (media synchronization, quality of perception, haptic rendering, degree of immersion, user satisfaction ) described in a linguistic scale and presented by different MFs, and the fuzzy logic infers the quality of experience using the output: intolerable, unacceptable, average, excellent, and perfect.

These studies inspire this work but we focus more on usermodeling for VR sickness. Therefore, this study aims to develop fuzzy models including FIS and ANFIS to predict the susceptibility to cybersickness arising from immersive virtual navigation based on the individual differences (e.g., age, gaming experience and ethnicity), enabling VR application designers to set navigation parameters that can provide user-customized experience. The novelties of this work are summarized as follows:

- Using a model-based FIS approach by integrating existing knowledge from the literature, we can have an overall understanding of the proneness to simulator sickness depending on explicit individual differences.

- Using a data-driven ANFIS approach, we can get rid of the literature knowledge but still extract knowledge from inputoutput datasets to predict an overall tendency to simulator sickness, which proved to have better performance than with the FIS approach.

- Our work opens the possibility to design adaptive VR applications based on individual characteristics.

\section{EfFECt OF Individual FACTORS ON CyBERSICKNESS}

Many individual factors can affect the susceptibility to cybersickness, but, as an exploratory study, we focused on Age, Gaming experience and Ethnicity at this stage, because we can obtain this information easily through questionnaires and fuzzify them straightforward through MFs. It should be noted that the correlation between these factors and cybersickness was summarized from the literature, and in this work, we accepted their findings as they were, which was a premise of our fuzzy logic model.

\subsection{Age}

Susceptibility to cybersickness rises up for children from the age of 2 to 12 years, but it remarkably decreases between 12 and 21 


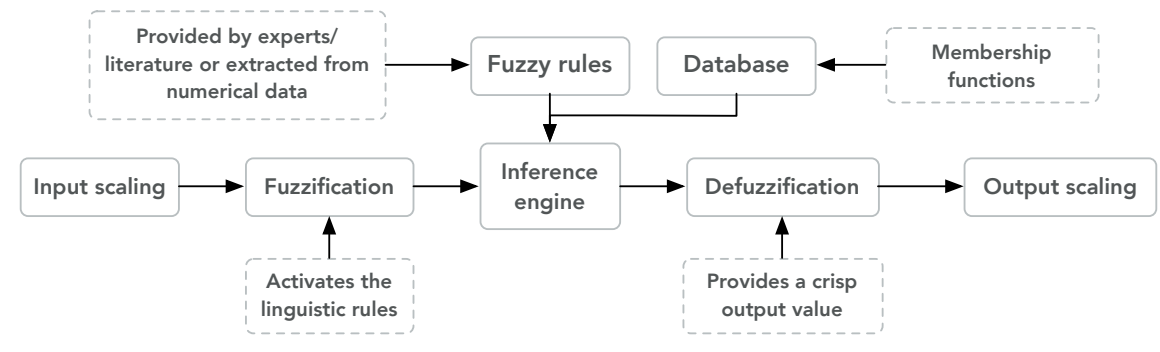

Figure 1: Block diagram for a fuzzy inference system.

years, then drops gradually thereafter $[25 \mid 35]$, as presented in Fig. 2 Therefore, the elder are less prone to cybersickness [7] and for those around 50, the occurrence of cybersickness is almost negligible [25]. Similarly, children prior to the age of 2 years are highly immune to cybersickness, which may have an evolutionary value as they are carried and jostled during this period [4]. Reason and Brand [35] explained such effect: children's senses are not fully formulated and are still highly flexible and adaptable.

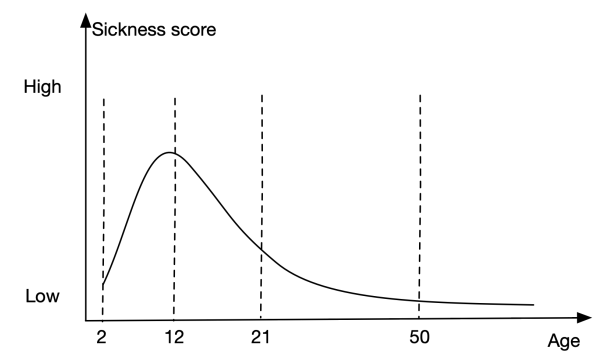

Figure 2: Trend of susceptibility to sickness against increasing age (inferred from [35] and [25]).

\subsection{Gaming experience}

Participants with prior gaming experience can perform better and interact with virtual environments more efficiently than those without, which implies that individual gaming experience can potentially affect some sickness symptoms. Smith et al. compared the performance in terms of spatial perception, task completion time, jumping ability, and travel speed and accuracy. They found that participants with a prior computer gaming experience gain an advantage over non-gamers, as gamers in $3 \mathrm{D}$ virtual environments are frequently requested to achieve rapid and accurate locomotion. They have developed solid abilities, including spatial awareness and navigation [38]. Similarly, Häkkinen et al. reported that participants with less computer proficiency, technology and gaming experience have significantly more cybersickness symptoms when exposed to mobile stereoscopic stimuli than other participants [15]. Furthermore, they suggested that participants who have little computer and gaming experience are likely to hold a more neutral or negative attitude rather than enthusiasm towards new technology, and therefore they will feel more stressful and pay excessive attention to sickness symptoms they are experiencing.

Knight and Arns claimed that young individuals are less prone to cybersickness due to a growing number of individuals with gaming experience [24], motivating us to investigate the effect of gaming experience on cybersickness. Iskenderova et al. performed a Pearson correlation to investigate the relationship between the frequency of playing games and cybersickness [16]. They found that individuals with more gaming experience report less side effects due to the habituation to cybersickness, since similar sickness symptoms may appear on non-VR displays. With the intention of using physiological signals to predict cybersickness, Dennison et al. also found that those with gaming experience generally report less cybersickness [8].

\subsection{Ethnicity}

Very little research investigates the effect of ethnicity on cybersickness, and to the best of our knowledge, one pioneer work was carried out by Klosterhalfen et al. [23]. In their experiment, 227 healthy Caucasian and 82 Asian subjects were exposed to nauseainducing body rotation on a rotational chair along the yaw axis. The motion-sickness susceptibility questionnaire (MSSQ) and the total rotation tolerance time (RT) measured the severity of sickness. The results showed that the average RT for the Caucasian group is significantly higher than for the Asian group, and the MSSQ score for the Caucasians is lower than for Asian participants. Reasons may be that Asian individuals probably inherited a lower threshold and sensitivity to the feedback on one or more physiological stimuli, including osmolality, stress hormones or blood pressure, which control the release of vasopressin [41]. A former study also found that Asian subjects suffer greater disturbance in gastric activity, and they reported more severe cybersickness than European-American and African-American groups when exposed to a rotating optokinetic drum to induce sickness [40].

\section{Model Architecture}

In this section, we briefly present the Sugeno-type ANFIS and Mamdani-type FIS methods. Their implementation is detailed clearly in the MATLAB fuzzy logic toolbox [1].

\subsection{Sugeno-type ANFIS architecture}

The architecture of ANFIS used in this study is illustrated in Fig. 3 . The system starts with three inputs, $x, y$ and $z$, representing Age, Gaming experience and Ethnicity, respectively. The MF $O_{1, i}$ implies the degree to which the given $t$ belongs to a quantifier, for example, little, middle and high. The MFs are minimum equal to 0 and maximum equal to 1 . Some MFs, such as triangular, trapezoidal and Gaussian, are qualified to use, but the most common is the Gaussian bell-shaped function, given as,

$$
M F(t)=\frac{1}{1+\left(\frac{t-c_{i}}{a_{i}}\right)^{2 b_{i}}}
$$

where $a_{i}, b_{i}$ and $c_{i}$ are the premise parameters to be optimized through the learning algorithm. When changing their values, the bell-shaped functions vary consequently, displaying various configurations of MFs.

Then the rule base embraces three fuzzy if-then rules of the Sugeno-type [42],

Rule 1: If $x$ is $A_{1}, y$ is $B_{1}$ and $z$ is $C_{1}$, then $f_{1}=p_{1} x+q_{1} y+$ $r_{1} z+s_{1}$

Rule 2: If $x$ is $A_{2}, y$ is $B_{2}$ and $z$ is $C_{2}$, then $f_{2}=p_{2} x+q_{2} y+$ $r_{2} z+s_{2}$ 


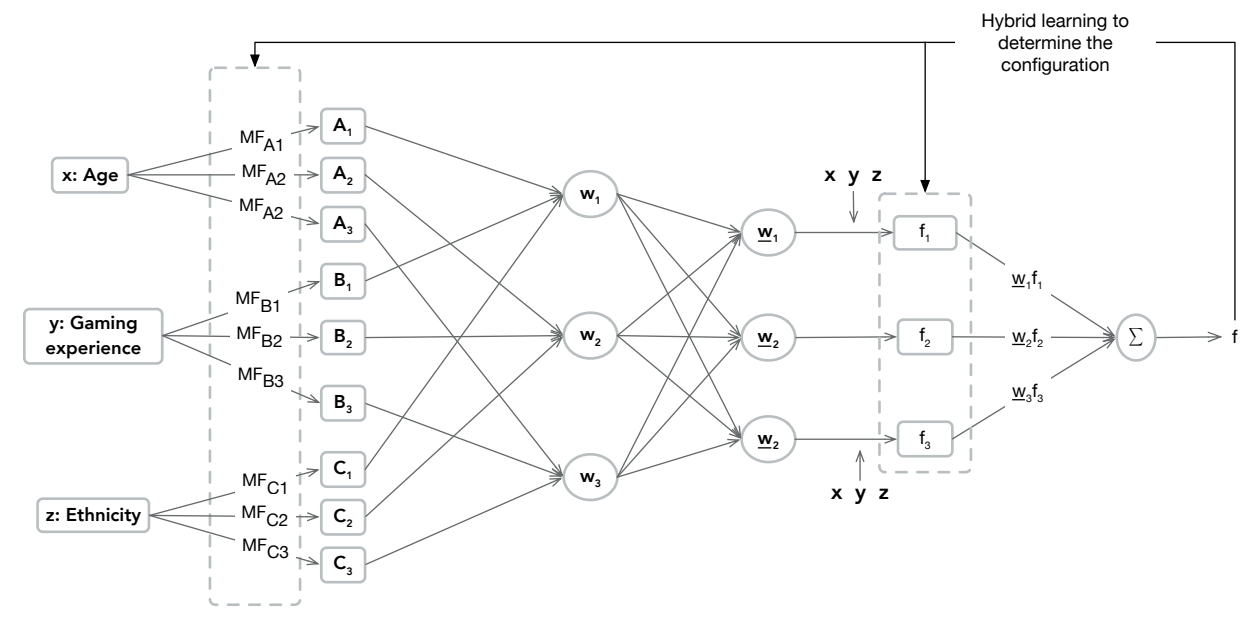

Figure 3: Adaptive neuro-fuzzy inference system architecture; note that the MFs and consequent functions are determined by expert knowledge in FIS, which is the main difference between ANFIS and FIS.

Rule 3: If $x$ is $A_{3}, y$ is $B_{3}$ and $z$ is $C_{3}$, then $f_{3}=p_{3} x+q_{3} y+$ $r_{3} z+s_{3}$

$M F_{A i}, M F_{B i}, M F_{C i}, f_{i}$ are parameterized functions, $i=1,2,3$. The purpose of the neural network is to adapt the premise parameters for $M F_{A i}, M F_{B i}, M F_{C i}$ and the consequent parameters $\left(p_{i}, q_{i}, r_{i}\right.$ and $s_{i}$ for $f_{i}$. The optimal settings of these parameters are searched through a hybrid learning algorithm to minimize the error between the observed and predicted outputs. The bell-shaped functions vary as the values change, displaying various configurations of MFs. A detailed explanation about the ANFIS architecture and related examples can be found in [5, 17, 48].

\subsection{Knowledge-based Mamdani-type FIS architecture}

In the ANFIS model (Fig. 3), the if-else rules should be constructed through the learning algorithm by adapting the parameters of the Gaussian bell-shaped function, while in the FIS model, instead of obtaining the configuration of the MFs and consequent functions through optimization, we can define them by summarizing from the existing literature directly. The consequent function in FIS is also represented by an MF. According to the above-mentioned effects of age, gaming experience and ethnicity in Section 3 and considering that in our database, participants were higher than 12 years old and included primarily Asian and European populations, we could design the following fuzzy if-else rules,

- - If the age is low, the level of cybersickness is high

- If the age is middle, the level of cybersickness is middle

- If the age is high, the level of cybersickness is low

- $\quad$ - If the gaming experience is little, the level of cybersickness is high

- If the gaming experience is middle, the level of cybersickness is middle

- If the gaming experience is high, the level of cybersickness is low

- - If the ethnicity is Asian, the level of cybersickness is high

- If the ethnicity is a miscegenation between European and Asian, the level of cybersickness is middle

- If the ethnicity is European, the level of cybersickness is low
FIS relies on these predefined fuzzy rules as premises to construct the inference system; thus, FIS is mainly employed in cases where either the system model is difficult to be constructed mathematically or the description of the studying problems is vague and equivocal. The Mamdani-type FIS is regarded as a knowledge-based system, since predefined if-else rules based on human knowledge from the experts or references are of great importance for this architecture. A detailed computing process is given in [18].

\subsection{Sampled data}

From our past studies (performed in the same condition) related to cybersickness, we accumulated 270 samples among which 26 samples were not included since the associated participants did not finish the test. Each sample consists of age, gaming experience, ethnicity, and cybersickness levels. Fig. 4 presents the individual characteristics of each sample from which we can observe the distribution. For the convenience of notation, the Ethnicity value of the Asians was assigned to 0 while that of the Caucasians was 1 . To our knowledge, we did not found any ethnic effects for participants from another ethnicity. Therefore, an Ethnicity value between 0 and 1 was selected considering their ancestors. Each sample's characteristics are scattered uniformly in the given range, which can help avoid data bias in the validation.

The experimental steps are illustrated in Fig. 5 First, the participants signed a consent form, and they were given instructions about what they were asked to do in the virtual environment and how to control their navigation with an HTC Vive hand controller or a Flystick controller in a CAVE system. Before the task started, participants had to fill a questionnaire about their health condition and information about age, gaming experience and ethnicity; they also had to fill the Simulator Sickness Questionnaire (SSQ) to record a pre-exposure level of cybersickness. They were allowed to drop out if they felt severe sickness. During the experiment, the participants navigated through the virtual scenario presented in Fig. 7 inside either an HTC Vive or a 5-sided 4K CAVE system, and using a controller-based navigation technique. All participants had to walk on the same path and navigate for an equal distance as fast as possible, during which they had to frequently accelerate, decelerate or make a turn to avoid obstacles on the path. The aim of these frequent changes in speed was to induce VR sickness easily. Each test lasted around 5.5 minutes on average. Because of the visual stimuli displayed to the participants, they could experience sickness symptoms. Hence, a second SSQ was requested to be filled after finishing the task in the immersive environment. The SSQ score 

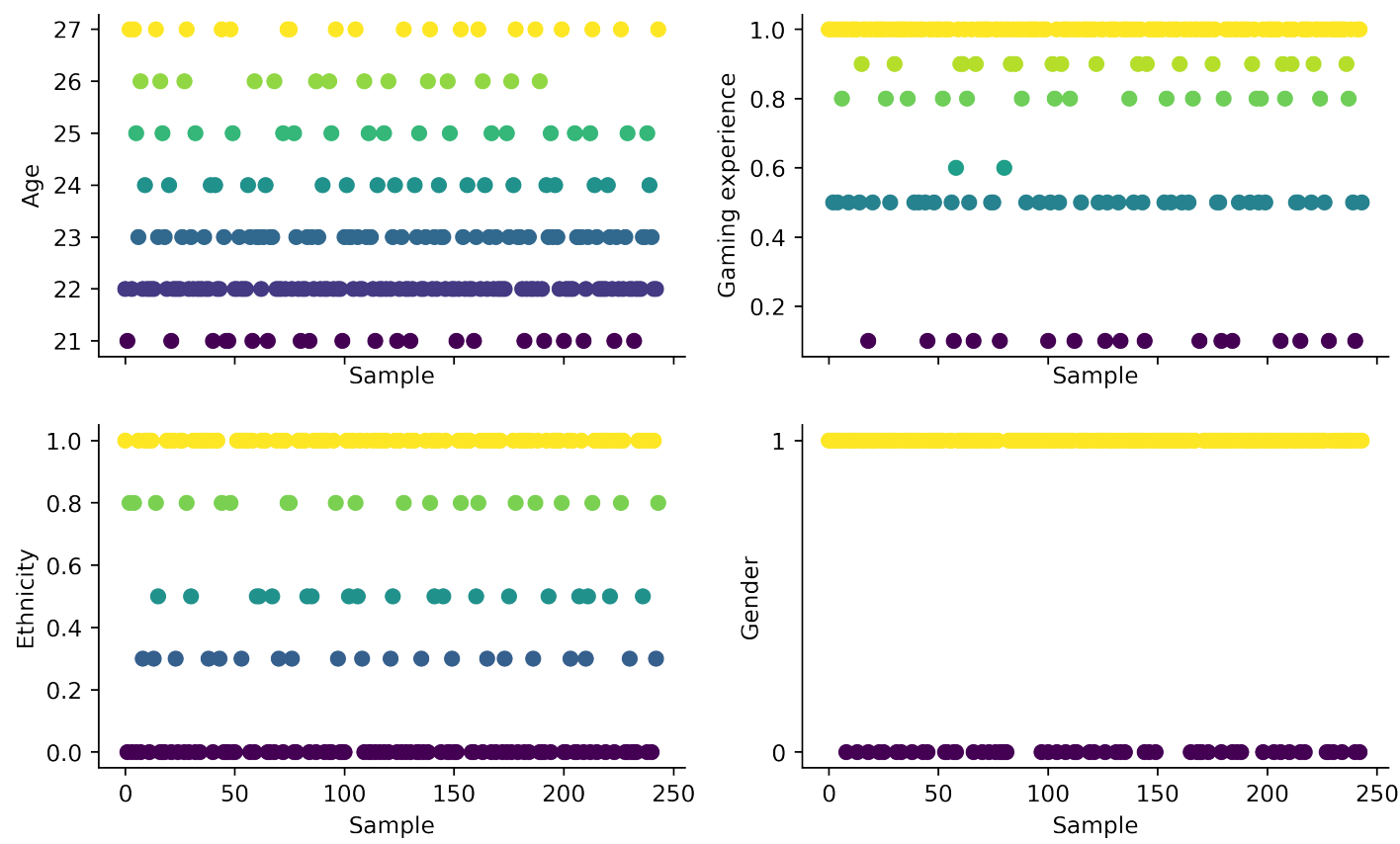

Figure 4: Individual characteristics of each sample, including Age, Gaming experience, Ethnicity and Gender.

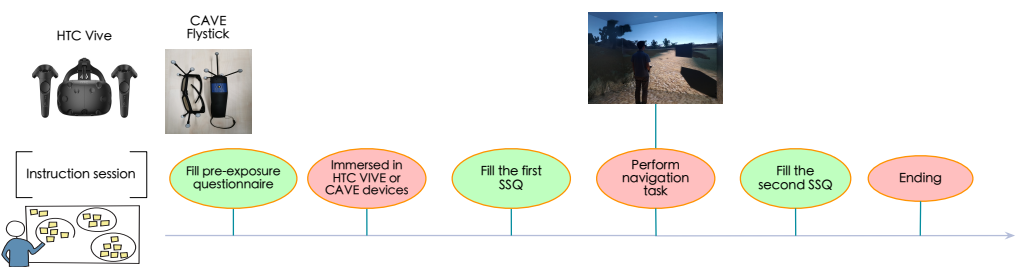

Figure 5: Experimental procedure conducted to collect data.

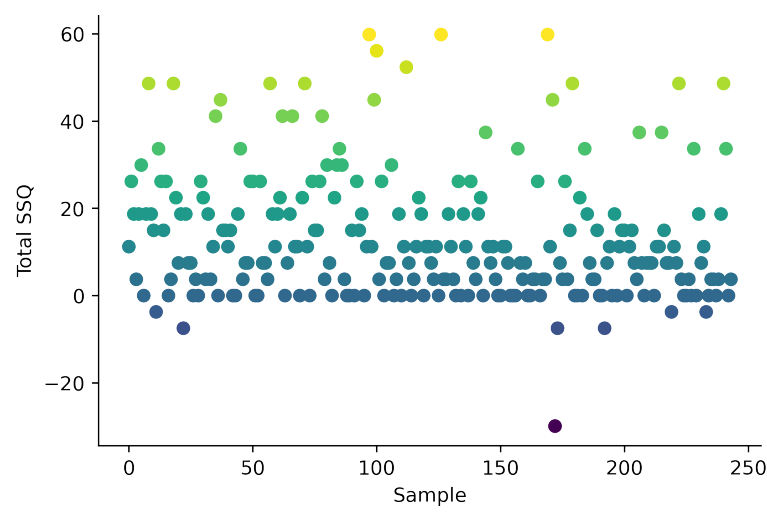

Figure 6: SSQ score with respect to each sample in Fig. 4

was measured as the difference between the post- and pre-exposure questionnaires. The distribution of the SSQ scores resulting from the experiment for each sample is depicted in Fig. 6

A preprocessing of the data was performed to convert the linguistic answers in the questionnaires to a crisp value that will constitute the input of the proposed fuzzy models. For example, "Caucasian" was assigned a value of 1 while "Asian" was assigned a value a 0 .

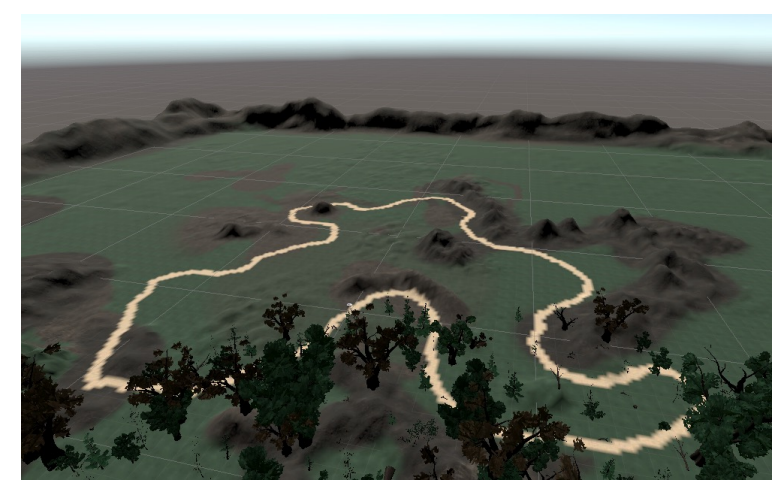

Figure 7: View of the virtual scenario in which the user had to navigate through the path.

\section{Validation of the Proposed Methods}

According to a given Age, Gaming experience and Ethnicity, FIS and ANFIS are supposed to predict the proneness to cybersickness, and we noted the proneness as $O_{\text {fis }}$ and $O_{\text {anfis }}$, respectively. During the validation, we expected to find an effective correlation between the predicted proneness and the ground truth simulator sickness questionnaire (SSQ) score. We made the following hypotheses,

- $O_{f i s}$ is correlated with the SSQ score 


\section{- $O_{\text {anfis }}$ is correlated with the SSQ score}

Note that we were interested in the SSQ score rather than its subscales (Nausea, Oculomotor, Disorientation) because we did not found enough expert knowledge from previous studies to link these sub-scale scores to individual characteristics.

\subsection{Principle of validation}

Our validation relied mainly on theoretical aspects at the current stage. Performance validations were carried out based on the correlation coefficient and a $k$-fold cross-validation. We will highlight the related concepts and explain why our architectures were reliable.

The correlation coefficient $r$ between two variables is represented by a value ranging from -1 to 1 , where a value of zero indicates the strongest disagreement and \pm 1 reveals a perfect fit and agreement [43]. With the correlation coefficient, we expected to see how much the output from fuzzy models can be associated with the SSQ, and if the fuzzy models worked well to composite individual differences, we would get a proper value of $r$ between the composite value and the SSQ.

The $k$-fold cross-validation [33] is a statistical method to assess the performance of machine learning models on unseen data. This model is easy to handle and implement with lower bias than other methods [2]. The procedure contains a single parameter $k$ indicating the number of sets that the assigned data is divided into around equal size. Every set is sequentially used to test the model constructed from $k-1$ training sets. Figure 8 shows the validation process for a $k=4$-fold cross-validation. In ANFIS, we implemented a 4-fold cross-validation method to validate the outputs' reliability: 3-fold of data for training the ANFIS model and the remaining fold of data for computing the correlation coefficient. In Mamdani-type FIS, since there is no training process, we computed each fold of data's correlation coefficient and took their average as the mean correlation coefficient.

\subsection{Correlation between Mamdani-type FIS outputs and SSQ}

Tab. 1 contains the correlation coefficients among individual factors, the level of cybersickness (i.e., the SSQ score) and the Mamdanitype FIS output (i.e., $O_{\text {fis }}$ ), and the p-value associated with each correlation coefficient aside to show the statistical power. If our assumption was true, the correlation between the SSQ score and individual differences should be congruent with the correlation between $O_{f i s}$ and individual differences. The significance level in this work was set to .05 .

A significant negative correlation was observed between $O_{f i s}$ and Ethnicity $\left(r_{59}=-.736, p<.01\right)$ and Gaming experience $\left(r_{59}=\right.$ $-.509, p<.01)$, while the correlation between $O_{f i s}$ and Age was very weak, $r_{59}=.215, p=.114$. The negative symbol validated the if-else rules for the effect of Ethnicity and Gaming experience, but the if-else rule for the effect of Age needed further investigation.

The SSQ score illustrated a similar trend as $O_{f i s}$ in terms of correlation with other factors, but only the correlation between SSQ and Gaming experience was significant, $r_{59}=-.367, p=.022$. The correlations between SSQ and Ethnicity $\left(r_{59}=-.238, p=\right.$ $.297)$, and between SSQ and Age $\left(r_{59}=-.048, p=.545\right)$ did not demonstrate any significance. Last, $O_{\text {fis }}$ and SSQ were found to be significantly correlated, $r_{59}=.438, p=.029$.

\subsection{Correlation between ANFIS outputs and SSQ}

Under the same statistical approach and data splitting, Tab.2 2 reports the correlation coefficients among individual factors, the SSQ score and the ANFIS output (i.e., $O_{\text {an fis }}$ ), and the corresponding p-value for each correlation coefficient. Since the correlation between SSQ and other individual factors has been reported in Tab. 11 these values did not change in Tab. 2. Therefore we focused on reporting the correlations between $O_{\text {anfis }}$ and the related individual factors.
A significant negative correlation was observed between $O_{\text {anfis }}$ and Ethnicity $\left(r_{59}=-.363, p<.01\right)$ and Gaming experience $\left(r_{59}=\right.$ $-.573, p<.01)$, while the correlation between $O_{a n f i s}$ and Age was not significant, $r_{59}=-.078, p=.596$. Similarly, $O_{\text {anfis }}$ was inversely correlated with Ethnicity and Gaming experience, which was also consistent with the respective if-else rules developed in the previous section. $O_{\text {anfis }}$ and SSQ were found to be better correlated compared with the Mamdani-type output, $r_{59}=.624, p<.01$.

\section{Discussion}

This study aimed to find a way to composite individual factors to predict the susceptibility to cybersickness, and we proposed our approach with the fuzzy logic. With predefined if-else rules and data learned features, the outputs of FIS and ANFIS demonstrated significant correlations with the SSQ. The FIS and ANFIS architectures were formulated differently, but it validated both architectures and the feasibility of our proposal that VR developers could use fuzzy logic to involve individual factors during the design of navigation interfaces. For example, depending on $O_{\text {fis }}$ or $O_{\text {anfis }}$ predicted from the user's characteristics, the navigation speed, acceleration, and immersion time could be adapted accordingly (Fig. 9).

Many individual factors can affect the proneness to cybersickness [27 44], but the models comprised only three factors (Ethnicity, Age and Gaming experience), which might be the reason why the correlation magnitude was moderate, .438 for the Mamdani-type FIS and .624 for ANFIS. Thanks to the scalability of FIS and ANFIS, we can easily introduce additional factors to characterize cybersickness more precisely. To introduce new factors into the Mamdani-type FIS, we needed to provide clearly the if-else rules for this factor. For example, Illness is another factor reported to increase the susceptibility to cybersickness [27]; thus, the if-else rules could be:

- If the participant is a little ill, the level of cybersickness is low

- If the participant is moderately ill, the level of cybersickness is middle

- If the participant is seriously ill, the level of cybersickness is high

Then, these new rules could be added to the Mamdani-type FIS architecture for estimating the level of cybersickness. Simultaneously, ANFIS can automatically construct the if-else rules based on the fed data. Depending on the situation, if we knew the potential effect of such factor on the susceptibility to cybersickness, we could apply the Mamdani-type FIS model; otherwise, the data-driven ANFIS could be an advantageous alternative. However, we cannot add an arbitrary factor to this model. Fuzzy logic is a computing method based on the "degree of truth" instead of the usual "true or false" (1 or 0) Boolean logic. The membership function expresses the degree of truth. For example, when participants are asked to report their gender, two choices are usually proposed in past studies, male or female, which is not fuzzy but Boolean logic.

As we had involved three human factors and an SSQ score in our dataset, the dataset reliability validation should find some correlations among these factors. However, only a correlation between SSQ and the Gaming experience reported from our dataset $\left(r_{59}=-0.367, p=.022\right)$ was close to the one reported in [16] $\left(r_{31}=-0.38, p=.04\right)$. The absence of significant correlations between SSQ and Ethnicity and between SSQ and Age might be due to the lack of enough participants with different ethnicity and ages. According to past literature [25 35], Age is supposed to be an influential factor that affects the level of cybersickness. However, ANFIS also did not find any significant effect of Age despite its learning ability, and such insignificance was also observed from the Mamdani-type FIS output. This finding needed to be interpreted with similar caution: the Age of the participants considered here 


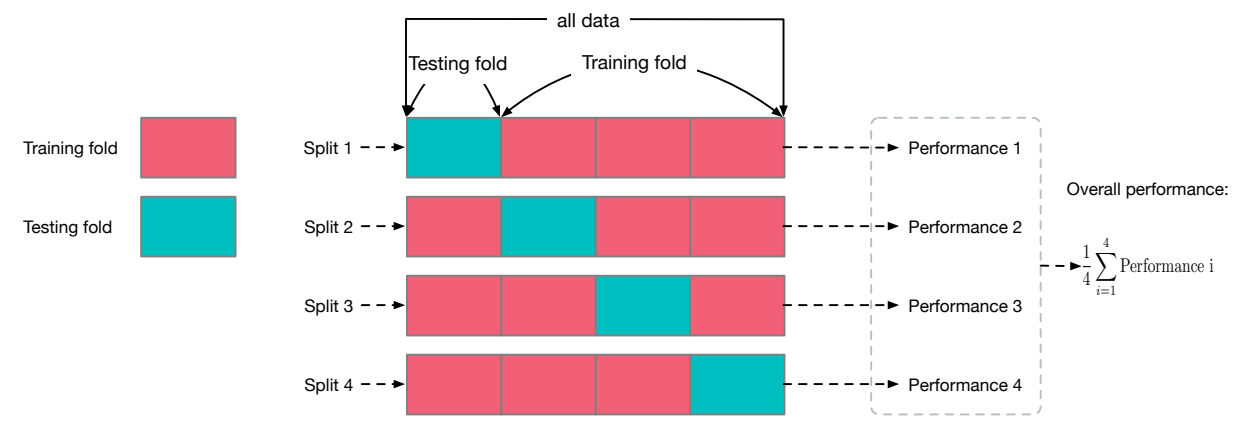

Figure 8: Example of a 4-fold cross validation: the data is split into four folds and in each division, the model is built with three folds and validated with one fold; the overall performance is obtained by computing the arithmetic mean.

Table 1: Correlation coefficients and p-values between inputs and Mamdani-type FIS outputs

\begin{tabular}{|c|c|c|c|c|c|c|c|c|c|c|}
\hline & \multicolumn{2}{|c|}{$O_{\text {fis }}$} & \multicolumn{2}{|c|}{ Ethnicity } & \multicolumn{2}{|c|}{ Age } & \multicolumn{2}{|c|}{ Gaming experience } & \multicolumn{2}{|c|}{ SSQ } \\
\hline & correlatior & p-value & correlation & p-value & correlation & p-value & correlatior & p-value & correlation & p-value \\
\hline$O_{f i s}$ & 1 & . & & $\ddots$ & $\ddots$. & $\ddots$. & $\ddots$. & $\ddots$ & $\ddots$. & . \\
\hline Ethnicity & -0.736 & 0.005 & 1 & $\cdot$ & $\cdot$ & $\ddots$ & $\ddots$ & $\ddots$ & $\ddots$ & . \\
\hline Age & 0.215 & 0.114 & -0.1626 & 0.359 & 1 & $\ddots$ & $\ddots$ & $\ddots$. & $\ddots$ & $\ddots$ \\
\hline Gaming experience & -0.509 & 0.001 & 0.030 & 0.439 & -0.371 & 0.009 & 1 & $\ddots$ & $\ddots$ & 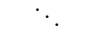 \\
\hline SSQ & 0.438 & 0.029 & -0.238 & 0.297 & -0.048 & 0.545 & -0.367 & 0.022 & 1 & $\ddots$ \\
\hline
\end{tabular}

Table 2: Correlation coefficients and p-values between inputs and ANFIS outputs

\begin{tabular}{|c|c|c|c|c|c|c|c|c|c|c|}
\hline & \multicolumn{2}{|c|}{$O_{\text {anfis }}$} & \multicolumn{2}{|c|}{ Ethnicity } & \multicolumn{2}{|c|}{ Age } & \multicolumn{2}{|c|}{ Gaming experience } & \multicolumn{2}{|c|}{ SSQ } \\
\hline & correlation & p-value & correlation & p-value & correlation & p-value & correlation & p-value & correlation & p-value \\
\hline$O_{\text {anfis }}$ & 1 & & & . & $\ddots$ & $\therefore$ & $\ddots$ & $\ddots$ & $\ddots$ & $\ddots$ \\
\hline Ethnicity & -0.365 & 0.005 & 1 & $\cdot$ & $\ddots$ & $\ddots$ & $\ddots$ & $\ddots$ & $\ddots$ & $\cdot$ \\
\hline Age & -0.078 & 0.596 & -0.163 & 0.359 & 1 & 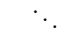 & $\ddots$ & $\ddots$. & $\ddots$ & $\cdot$ \\
\hline Gaming experience & -0.573 & 0.001 & 0.030 & 0.439 & -0.371 & 0.009 & 1 & $\ddots$ & $\ddots$ & $\ddots$ \\
\hline SSQ & 0.624 & 0.001 & -0.238 & 0.297 & -0.048 & 0.545 & -0.367 & 0.022 & 1 & 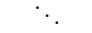 \\
\hline
\end{tabular}

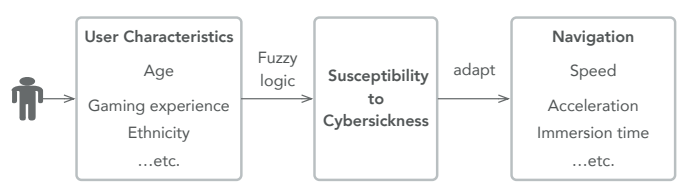

Figure 9: Process for using fuzzy logic methods to predict user's susceptibility to cybersickness and then adapt navigation parameters.

ranged from 18 to 35 , which only covered a small range of age, and a further study with participants from the adolescent to the elderly might provide more substantial support. As a cybersickness predictor, $O_{\text {fis }}$ or $O_{\text {anfis }}$ were significantly correlated with Ethnicity and Gaming experience whereas SSQ was not. Reasons might be that the SSQ value from the questionnaires could contain unreliable, ambiguous and arbitrary emotional feedback [21]. Instead, $O_{\text {fis }}$ or $O_{\text {anfis }}$ derived from either Mamdani-type FIS or ANFIS can avoid noisy information and strongly link to both sides (SSQ and individual factors).

We presented two strategies to integrate individual factors to propose a cybersickness indicator. With existing knowledge about the effect of personal characteristics, FIS could alleviate the amount of data needed to produce a successful estimation of cybersickness, keeping the consumption of data to a minimum, as data are expensive to produce and obtain. On the other hand, with the available data, ANFIS proved to have better performance than FIS because ANFIS can learn and extract features from numerical data. Theoretically, artificial neural network (ANN) or other regression approaches can also learn the feature actively from the data to associate individual attributes with the level of cybersickness. Additionally, there are many studies comparing their performance, suggesting that both approaches could present competitive achievement if fine-tuned [22. 48. However, this paper aimed to engage individual differences into VR navigation systems. We preferred to use the FIS method because it can utilize prior expert knowledge naturally without using any data. It was also important to distinguish between a regression problem and a fuzzy logic problem: a regression problem aims to find the unknown correlation among variables, but the fuzzy logic method will take advantage of existing correlation (i.e., expert knowledge) for prediction. In our work, we had known the effect of Age, Gaming experience and Ethnicity on cybersickness from literature, and we composited such knowledge through fuzzy logic and provided a single output. Hence, we did not use the regression method as the baseline to compare.

\section{Conclusion}

We proposed fuzzy logic as a method to composite existing knowledge about the effect of individual factors on cybersickness, and we successfully demonstrated a significant correlation between the composited value and the level of cybersickness. We only introduced three factors as individual properties (age, ethnicity, and gaming experience) in this study, which was not enough to cover all individual differences in VR sickness susceptibility when exposing users to 
visual stimuli. Put differently, the correlation coefficient's magnitude was moderate, probably due to the lack of involved individual factors. Still, significant $p$-values ensured the reliability of the output of fuzzy logic despite small correlation coefficients. Hence, further factors such as gender, controllability of postural stability, physical illness, visual acuity and emotional states were worth investigating with the proposed approach, which shall enhance the precision and reliability of the model. However, including too many factors may introduce additional noise to the model, as their effects on VR sickness were not apparent. We would perform a deeper literature study, experiment and develop some pre-processing techniques for these factors to improve the overall reliability.

Thanks to fuzzy logic, we can exploit the premise knowledge from experts, literature and data to predict side effects individually. In the next step, we were planning to carry out user experiments on a VR platform in which the navigation interface can be adapted according to the output of fuzzy logic, and we will also consider involving some real-time adaptation strategy based on physiological signals (e.g., GSR, HRV), allowing us to develop an immersive VR environment with better customized performance and experience.

\section{ACKNOWLEDGMENTS}

This work was supported by the China Scholarship Council (201708390014), the French-German Doctoral School (CDFA-0319), and the Erasmus+ mobility scholarship of HESAM.

\section{REFERENCES}

[1] Fuzzy Logic Toolbox: User's Guide (R2020a). Natick, Massachusetts, United State, 2020. The MathWorks, Inc.

[2] Y. Bengio and Y. Grandvalet. No unbiased estimator of the variance of k-fold cross-validation. Journal of machine learning research, 5(Sep):1089-1105, 2004.

[3] K. Benmouiza and A. Cheknane. Clustered ANFIS network using fuzzy c-means, subtractive clustering, and grid partitioning for hourly solar radiation forecasting. Theoretical and Applied Climatology, 137(12):31-43, 2019. doi: 10.1007/s00704-018-2576-4

[4] F. Biocca. Will simulation sickness slow down the diffusion of virtual environment technology? Presence: Teleoper. Virtual Environ., 1(3):334-343, Jan. 1992. doi: 10.1162/pres.1992.1.3.334

[5] Y.-S. Chen, C.-H. Cheng, C.-L. Chiu, and S.-T. Huang. A study of anfis-based multi-factor time series models for forecasting stock index Applied Intelligence, 45(2):277-292, 2016. doi: 10.1007/s10489-016 $-0760-8$

[6] G. Cueva-Fernandez, J. P. Espada, V. García-Díaz, R. G. Crespo, and N. Garcia-Fernandez. Fuzzy system to adapt web voice interfaces dynamically in a vehicle sensor tracking application definition. Soft Computing, 20(8):3321-3334, 2016. doi: 10.1007/s00500-015-1709-2

[7] S. Davis, K. Nesbitt, and E. Nalivaiko. A Systematic Review of Cybersickness. In Proceedings of the 2014 Conference on Interactive Entertainment - IE2014, pp. 1-9. ACM Press, New York, New York, USA, 2014. doi: 10.1145/2677758.2677780

[8] M. S. Dennison, A. Z. Wisti, and M. D'Zmura. Use of physiological signals to predict cybersickness. Displays, 44:42-52, sep 2016. doi: 10 .1016/j.displa.2016.07.002

[9] N. Duzmanska, P. Strojny, and A. Strojny. Can simulator sickness be avoided? A review on temporal aspects of simulator sickness. Frontiers in Psychology, 9(NOV), 2018. doi: 10.3389/fpsyg. 2018.02132

[10] S. Gibbons. How businesses are using vr to survive the covid-19 era. Online, may 2020.

[11] J. F. Golding. Motion sickness susceptibility questionnaire revised and its relationship to other forms of sickness. Brain research bulletin, 47(5):507-516, 1998. doi: 10.1016/S0361-9230(98)00091-4

[12] M. K. Goyal, B. Bharti, J. Quilty, J. Adamowski, and A. Pandey. Modeling of daily pan evaporation in sub tropical climates using ann, ls-svr, fuzzy logic, and anfis. Expert systems with applications, 41(11):52675276, 2014. doi: 10.1016/j.eswa.2014.02.047

[13] A. Hamam, M. Eid, A. El Saddik, and N. D. Georganas. A fuzzy logic system for evaluating quality of experience of haptic-based applications.
In International Conference on Human Haptic Sensing and Touch Enabled Computer Applications, pp. 129-138. Springer, 2008. doi: 10. 1007/978-3-540-69057-3_14

[14] F. Hu, Y. Deng, W. Saad, M. Bennis, and A. H. Aghvami. Cellularconnected wireless virtual reality: Requirements, challenges, and solutions, 2020.

[15] J. Häkkinen, M. Liinasuo, J. Takatalo, and G. Nyman. Visual comfort with mobile stereoscopic gaming. In A. J. Woods, N. A. Dodgson, J. O. Merritt, M. T. Bolas, and I. E. McDowall, eds., Stereoscopic Displays and Virtual Reality Systems XIII, vol. 6055, pp. 85 - 93. International Society for Optics and Photonics, SPIE, 2006. doi: 10.1117/12.641210

[16] A. Iskenderova, F. Weidner, and W. Broll. Drunk Virtual Reality Gaming:Exploring the Influence of Alcohol on Cybersickness. In Proceedings of the Annual Symposium on Computer-Human Interaction in Play - CHI PLAY'17, pp. 561-572. ACM Press, New York, New York, USA, 2017. doi: 10.1145/3116595.3116618

[17] J.-S. Jang. Anfis: adaptive-network-based fuzzy inference system. IEEE transactions on systems, man, and cybernetics, 23(3):665-685, 1993. doi: 10.1109/21.256541

[18] J.-S. R. Jang and C.-T. Sun. Neuro-Fuzzy and Soft Computing: A Computational Approach to Learning and Machine Intelligence. PrenticeHall, Inc., USA, 1996.

[19] M. Javaid and A. Haleem. Virtual reality applications toward medical field. Clinical Epidemiology and Global Health, 2019. doi: 10.1016/j. cegh.2019.12.010

[20] D. M. Johnson. Introduction to and review of simulator sickness research. Technical report, ARMY RESEARCH INST FIELD UNIT FORT RUCKER AL, 2005

[21] J. Katicic, P. Häfner, and J. Ovtcharova. Methodology for Emotional Assessment of Product Design by Customers in Virtual Reality. Presence: Teleoperators and Virtual Environments, 24(1):62-73, feb 2015. doi: 10.1162/PRES_a_00215

[22] B. Khoshnevisan, S. Rafiee, M. Omid, and H. Mousazadeh. Development of an intelligent system based on ANFIS for predicting wheat grain yield on the basis of energy inputs. Information Processing in Agriculture, 1(1):14-22, 2014. doi: 10.1016/j.inpa.2014.04.001

[23] S. Klosterhalfen, S. Kellermann, F. Pan, U. Stockhorst, G. Hall, and P. Enck. Effects of ethnicity and gender on motion sickness susceptibility. Aviation Space and Environmental Medicine, 76(11):1051-1057, 2005.

[24] M. M. Knight and L. L. Arns. The relationship among age and other factors on incidence of cybersickness in immersive environment users. In ACM SIGGRAPH 2006 Research Posters, SIGGRAPH '06. ACM, New York, NY, USA, 2006. doi: 10.1145/1179622.1179846

[25] E. M. Kolasinski. Simulator sickness in virtual environments. Technical report, Army Research Inst for the Behavioral and Social Sciences Alexandria Va, 1995.

[26] A. Kulik. Building on realism and magic for designing 3d interaction techniques. IEEE Computer Graphics and Applications, 29(6):22-33, nov 2009. doi: 10.1109/MCG. 2009.115

[27] J. J. LaViola Jr. A discussion of cybersickness in virtual environments. ACM Sigchi Bulletin, 32(1):47-56, 2000.

[28] F. Merienne. Virtual Reality: Principles and Applications. In Encyclopedia of Computer Science and Technology, Second Edition, vol. 2017, pp. 1-11. CRC Press, dec 2016. doi: 10.1081/E-ECST2-140000194

[29] NAE. Nae grand challenges for engineering. Technical report, National Academy of Engineering, 2017.

[30] J. Negrillo-Cárdenas, J.-R. Jiménez-Pérez, and F. R. Feito. The role of virtual and augmented reality in orthopedic trauma surgery: From diagnosis to rehabilitation. Computer Methods and Programs in Biomedicine, 191:105407, 2020. doi: 10.1016/j.cmpb.2020.105407

[31] H. O. Nyongesa, T. Shicheng, S. Maleki-Dizaji, S.-T. Huang, and J. Siddiqi. Adaptive web interface design using fuzzy logic. In Proceedings IEEE/WIC International Conference on Web Intelligence (WI 2003), pp. 671-674. IEEE, 2003. doi: 10.1109/WI.2003.1241293

[32] D. Ota, B. Loftin, T. Saito, R. Lea, and J. Keller. Virtual reality in surgical education. Computers in Biology and Medicine, 25(2):127 137, 1995. Virtual Reality for Medicine. doi: 10.1016/0010-4825(94) 00009-F

[33] R. R. Picard and R. D. Cook. Cross-validation of regression models. 
Journal of the American Statistical Association, 79(387):575-583, 1984. doi: 10.1080/01621459.1984.10478083

[34] J. Radianti, T. A. Majchrzak, J. Fromm, and I. Wohlgenannt. A systematic review of immersive virtual reality applications for higher education: Design elements, lessons learned, and research agenda. Computers \& Education, 147:103778, 2020. doi: 10.1016/j.compedu. 2019.103778

[35] J. T. Reason and J. J. Brand. Motion sickness. Academic press, 1975.

[36] L. Rebenitsch and C. Owen. Individual variation in susceptibility to cybersickness. In Proceedings of the 27th annual ACM symposium on User interface software and technology - UIST '14, pp. 309-317. ACM Press, New York, New York, USA, 2014. doi: 10.1145/2642918. 2647394

[37] W. R. Sherman and A. B. Craig. Understanding Virtual Reality: Interface, Application, and Design. Morgan Kaufmann Publishers Inc., 2003.

[38] S. P. Smith and S. Du'Mont. Measuring the effect of gaming experience on virtual environment navigation tasks. In 2009 IEEE Symposium on 3D User Interfaces, pp. 3-10. IEEE, 2009. doi: 10.1109/3DUI.2009. 4811198

[39] P. Srisaeng, G. S. Baxter, and G. Wild. An adaptive neuro-fuzzy inference system for forecasting australia's domestic low cost carrier passenger demand. Aviation, 19(3):150-163, 2015. doi: 10.3846/ 16487788.2015.1104806

[40] R. Stern, S. Hu, R. LeBlanc, and K. Koch. Chinese hyper-susceptibility to vection-induced motion sickness. Aviation, space, and environmental medicine, 64(9 Pt 1):827-830, September 1993.

[41] R. M. Stern, S. Hu, S. H. Uijtdehaage, E. R. Muth, L. H. Xu, and K. L. Koch. Asian hypersusceptibility to motion sickness. Human heredity, 46(1):7-14, 1996. doi: 10.1159/000154318

[42] T. Takagi and M. Sugeno. Derivation of fuzzy control rules from human operator's control actions. IFAC Proceedings Volumes, 16(13):55-60, 1983. doi: 10.1016/S1474-6670(17)62005-6

[43] R. Taylor. Interpretation of the Correlation Coefficient: A Basic Review. Journal of Diagnostic Medical Sonography, 6(1):35-39, jan 1990. doi: $10.1177 / 875647939000600106$

[44] S. Weech, S. Kenny, and M. Barnett-Cowan. Presence and Cybersickness in Virtual Reality Are Negatively Related: A Review. Frontiers in Psychology, 10(FEB):1-19, feb 2019. doi: 10.3389/fpsyg.2019.00158

[45] D. Wu, C. G. Courtney, B. J. Lance, S. S. Narayanan, M. E. Dawson, K. S. Oie, and T. D. Parsons. Optimal arousal identification and classification for affective computing using physiological signals: Virtual reality stroop task. IEEE Transactions on Affective Computing, 1(2):109-118, jul 2010. doi: 10.1109/T-AFFC.2010.12

[46] G.-Z. Yang, B. J. Nelson, R. R. Murphy, H. Choset, H. Christensen, S. H. Collins, P. Dario, K. Goldberg, K. Ikuta, N. Jacobstein, D. Kragic, R. H. Taylor, and M. McNutt. Combating covid-19-the role of robotics in managing public health and infectious diseases. Science Robotics, 5(40), 2020. doi: 10.1126/scirobotics.abb5589

[47] Z. Yang, Y. Liu, and C. Li. Interpolation of missing wind data based on anfis. Renewable Energy, 36(3):993-998, 2011. doi: 10.1016/j.renene. 2010.08.033

[48] M. Şahin and R. Erol. A Comparative Study of Neural Networks and ANFIS for Forecasting Attendance Rate of Soccer Games. Mathematical and Computational Applications, 22(4):43, 2017. doi: 10. 3390/mca22040043 